\title{
High-dose-rate vaginal brachytherapy with chemotherapy for surgically staged localized uterine serous carcinoma
}

\author{
Shari Damast, MD!, Susan A. Higgins, MD!, Elena Ratner, MD², Maria C. De Leon, MD², Sheida Mani, MD!, \\ Dan-Arin Silasi, MD², Masoud Azodi, MD², Alessandro Santin, MD², Thomas Rutherford, MD, PhD², \\ Peter E. Schwartz, MD² \\ 'Department of Therapeutic Radiology. 2Department of Obstetrics, Gynecology, and Reproductive Sciences, Division of Gynecologic \\ Oncology, Yale University School of Medicine, New Haven, CT, USA
}

\begin{abstract}
Purpose: To evaluate our institutional experience combining carboplatin-paclitaxel $(\mathrm{C} / \mathrm{T})$ chemotherapy with high-dose-rate (HDR) intra-vaginal brachytherapy (IVB) following comprehensive surgical staging in localized uterine serous carcinoma (USC).

Material and methods: Institutional chart review identified 56 patients with FIGO 2009 stage I-II USC treated between 2000-2010. Patients underwent total hysterectomy, bilateral salpingo-oopherectomy, and comprehensive surgical staging including pelvic and para-aortic lymph node dissection, omentectomy, and peritoneal cytology. Chemotherapy was 6 cycles of C/T, and the IVB dose was $14 \mathrm{~Gy}$ in 2 fractions, prescribed to $0.5 \mathrm{~cm}$ from the cylinder surface. Kaplan-Meier methods were used to estimate recurrence-free survival (RFS) and overall survival (OS).

Results: The median follow-up time was 49 months (range: 9-145). The 5-yr RFS and OS were 85\% and 93\%, respectively. In all cases of recurrence $(n=8)$, the first site of failure was extra-pelvic. There were no isolated vaginal recurrences, however, there was one vaginal apex recurrence recorded at 19 months in a patient with simultaneous lung metastases. Thus, the 2-year vaginal RFS was $98 \%$.

Conclusions: Excellent vaginal/pelvic control rates were observed. Further study of HDR brachytherapy dose and fractionation in combination with chemotherapy is worthwhile.

Key words: brachytherapy, chemotherapy, endometrial cancer, papillary serous.

\section{Purpose}

Uterine serous carcinoma (USC) is an uncommon but aggressive form of endometrial adenocarcinoma [1]. Its pattern of spread favors the peritoneum and upper abdomen, and thus adjuvant management is typically more aggressive than for the endometrioid subtypes, even when it presents in early stages [2]. In the United States, surgical staging of USC includes total hysterectomy, bilateral salpingo-oophorectomy, peritoneal washings, omentectomy, and pelvic and para-aortic lymph node dissections [2]. Historically, whole abdominal radiotherapy was the mainstay of adjuvant therapy, even in optimally resected stage I disease $[3,4]$. Recently, studies have demonstrated a benefit with the use of adjuvant chemotherapy [5-7]. Contemporary adjuvant treatment of early stage (stage I-II) disease following comprehensive staging involves a combination of chemotherapy and smaller-field radia- tion therapy (RT) [5,6], and an increasingly popular adjuvant treatment paradigm combines chemotherapy with intra-vaginal brachytherapy (IVB) [5,8-13].

There have been few studies to date, which have addressed adjuvant IVB technique in early stage USC, and thus questions concerning IVB dose and sequencing with chemotherapy persist. This topic was not covered in the guidelines for adjuvant vaginal cuff brachytherapy released by the American Brachytherapy Society in 2012 [14]. Several recent institutional reports describe excellent 5 -year vaginal recurrence rates of $0-3 \%$ using chemotherapy in combination with a common high-dose-rate (HDR) IVB regimen, 7 Gy $\times 3$ fractions prescribed to a depth of $5 \mathrm{~mm}$ from applicator surface [9-12]. A recent phase II evaluation of endometrial patients with high-intermediate risk factors, of which $35 \%$ of the population was USC, employed this same HDR regimen followed by three cycles of adjuvant chemotherapy [15]. There is modest sup-
Address for correspondence: Shari Damast, MD, Department of Therapeutic Radiology, Yale University School of Medicine, 15 York St New Haven, CT 06510, USA, phone: +1 203-200-2635, fax: +1 203-785-4622, • e-mail: shari.damast@yale.edu
Received: 10.09 .2014

Accepted: 21.11 .2014

Published: 28.02.2015 
port for an alternate IVB dose schedule for USC patients receiving chemotherapy based on a single retrospective report, in which HDR IVB was delivered twice per week in 6 fractions of 4 Gy prescribed to the mucosal surface [13]. This fractionation schedule delivered an equivalent dose in 2 Gy / fraction (EQD2) [16] of approximately 28 Gy to the vaginal surface, which is approximately half of the mucosal dose used in the other series.

At our institution, USC patients with localized disease who have undergone comprehensive surgical staging are recommended chemotherapy with IVB. When our HDR program was first introduced in the 1990s, in the era of adriamycin-based chemotherapy, several cases of vaginal mucositis were noted by the treating physicians, and thus the HDR prescription for all USC patients receiving chemotherapy was modified from 7 Gy $\times 3$ to 7 Gy $\times 2$ prescribed to $0.5 \mathrm{~cm}$ from the cylinder surface. This lower dose prescription translates into a total equivalent dose (EQD2) of approximately 36 Gy to the vaginal mucosal surface. Previously published results from our institution have demonstrated that this regimen appeared safe and effective [8], and resulted in significantly improved vaginal control when compared to outcomes of patients with localized USC who received chemotherapy alone [5].

Chemotherapy for USC has since evolved, and for the past 15 years, six cycles of adjuvant carboplatin-paclitaxel $(\mathrm{C} / \mathrm{T})$ chemotherapy has become standard. The IVB prescription dose at our institution for patients treated with this chemotherapy regimen has remained 2 fractions of 7 Gy prescribed at $0.5 \mathrm{~cm}$ depth. In this report, we present the 5-year clinical outcomes of patients with early stage (FIGO 2009 Stage I-II) USC treated at our institution from 2000-2010 with contemporary C/T chemotherapy and this unique brachytherapy regimen.

\section{Material and methods}

Institutional Review Board approval was granted to perform a retrospective chart review of all patients with stage I-II USC treated at our institution in the years 20002010. Patients with advanced-stage disease, or those with early stage disease who had received single modality therapy, external beam RT, other brachytherapy fractionation, or chemotherapy regimen other than $\mathrm{C} / \mathrm{T}$ were excluded. Additionally, patients with biopsy-proven cancer but without residual cancer in their hysterectomy specimens were excluded. Analysis was limited to patients who received all aspects of their multi-modal treatment at our academic medical center.

All patients had biopsy-proven endometrial cancer and underwent total hysterectomy with surgical staging. Pathology was reviewed by the institution's gynecologic pathologists prior to surgery. Chemotherapy consisted of carboplatin (AUC $=5-6)$ and paclitaxel $\left(175 \mathrm{mg} / \mathrm{m}^{2}\right)$ in all patients, given every 3 weeks for 6 cycles. Chemotherapy was typically initiated within 4-6 weeks from the time of surgery.

Intra-vaginal brachytherapy was delivered via a highdose-rate Ir-192 source. Patients were fit with a vaginal cylinder (size ranging from $2.6-3.0 \mathrm{~cm}$ ) at the time of radiation consultation. A dose of $14 \mathrm{~Gy}$ in 2 fractions (7 Gy each) was prescribed to a depth of $0.5 \mathrm{~cm}$ from the vaginal cylinder surface. Active source length was standardized for all patients to six dwell positions, separated by $1 \mathrm{~cm}$ spacing, for an active length of $5 \mathrm{~cm}$. All plans were created on a phantom with a $10 \mathrm{Ci}$ nominal source, and dwell times were optimized to ensure a dose of roughly 10.5-11 Gy at the cylinder surface. CT-simulation was not performed, and normal tissue point doses were not recorded.

Intra-vaginal brachytherapy was started early in the course of adjuvant treatment, typically with the first fraction delivered one week prior to and the second fraction one week following the second cycle of chemotherapy. Administering chemotherapy the same week as brachytherapy was avoided to decrease likelihood of overlapping toxicities. Patients were followed by routine clinical examination, Pap smears, and tumor markers every three months. Recurrences were identified radiographically or confirmed by biopsy. Based on review of physician-recorded toxicities listed in routine follow-up documentation, toxicity scores were assigned retrospectively with the Common Terminology Criteria for Adverse Events (CTCAE) v4.0.

Kaplan-Meier methods were used to estimate 5-year recurrence-free survival (RFS) and overall survival (OS). Time to recurrence, last follow-up, and/ or death was calculated from the date of hysterectomy. Log-rank test was employed to identify patient, tumor, and treatment characteristics associated with RFS and OS. Given the small sample size and paucity of events, a multivariate analysis was not performed. IBM SPSS Statistics v19.0 was used for all statistical analyses.

\section{Results}

Fifty-six patients were identified with FIGO 2009 stage I-II USC treated from 2000-2010 with adjuvant C/T chemotherapy along with 2 fractions of IVB (7 Gy each). The majority had undergone comprehensive surgical staging including pelvic dissection $(96 \%$, median nodes removed $=18$, range: $6-47$, para-aortic dissection $(80 \%$, median nodes removed $=3$, range: $1-37$ ), omentectomy, and peritoneal cytology (93\%). The median age was 66 (range: 47-81). According to FIGO 2009 staging, 42 patients $(73 \%)$ were stage IA, $18 \%$ were stage IB, and $9 \%$ were stage II. Of the IA patients, $14(33 \%)$ had disease confined to the endometrium, and 28 (67\%) had IA invasive disease. Among the entire cohort, $21 \%$ had myometrial invasion $\geq 50 \%$, and $29 \%$ had lymph-vascular space involvement. Forty percent of tumors consisted of USC mixed (more than 10\% USC) with other endometrioid or high grade histologies. Cytology was positive in 2 patients $(4 \%)$. Patient and tumor characteristics are detailed in Table 1.

All 56 patients completed 2 IVB fractions, and 53 patients $(95 \%)$ completed all 6 cycles of chemotherapy. The median time from surgery to first IVB fraction was 49 days (range: $30-113$ ). The median follow-up time, calculated from the date of hysterectomy, was 49 months (range: 9-145). Acute toxicities were mild. Late toxicities (recorded $>90$ days post-treatment) were as follows: forty-seven 
patients $(84 \%)$ had no toxicities recorded. Three patients with disease recurrence had grade 2-3 gastrointestinal toxicities. One patient who was disease-free with a history of ulcerative colitis had grade 2 diarrhea and tenesmus. Two patients had grade 2 neuropathy, one patient had grade 2 phlebitis. One patient had grade 2 urinary incontinence. Two patients (3.6\%) had grade 2 vaginal toxicities; one developed grade 2 vaginal stricture with banding, one was noted to have grade 2 vaginal atrophy.

Median survival time was not reached. The 5-year RFS and OS were $85 \%$ and $93 \%$, respectively. Kaplan-Meier curves are shown in Figure 1 and Figure 2. Univariate results are shown in Table 2 . Smoking history was associated with poorer OS $(p=0.008)$ and LVI (Lymph-vascular space invasion) was associated with poorer RFS $(p=0.003)$. Age $>70$ had a borderline significant effect on OS $(p=0.062)$ and smoking had a borderline effect on RFS $(p=0.076)$. Depth of myometrial invasion $\geq 50 \%$ was significantly associated with both poorer RFS $(p<0.0001)$ and OS $(p=0.001)$. Among patients with $\geq 50 \%$ myometrial invasion, the 5 -year RFS and OS was $47 \%$ and $72 \%$, versus $94 \%$ and $97 \%$ for those with $<50 \%$ myometrial invasion.

The percentage of patients with recurrence was $14.3 \%$ ( $n=8$ patients). In all cases, the first site of failure was extra-pelvic. The median time to recurrence was 28.5 months (range: 16-69). Neither of the two patients with positive peritoneal cytology at diagnosis (old FIGO 1988 stage IIIA) developed recurrence. One patient with no myometrial invasion (old FIGO 1988 stage IA) developed recurrence. Sites of recurrence are shown in Table 3. The most common failure site was lung. The para-aortic region was involved in 2 patients and was an isolated failure site in 1 patient. There were no pelvic recurrences. There were no isolated vaginal recurrences, however, there was one vaginal apex recurrence at 19 months in a patient with simultaneous lung metastases. Thus, the 2-year vaginal RFS was $98 \%$.

\section{Discussion}

Data concerning optimal IVB dose-fractionation in combination with chemotherapy for USC are lacking. The present report of 56 patients with surgically staged localized USC found that following aggressive surgery, an IVB dose of $14 \mathrm{~Gy}$ in two fractions prescribed to $5 \mathrm{~mm}$ depth (surface EQD2 36 Gy) in combination with 6 cycles of $\mathrm{C} / \mathrm{T}$ chemotherapy resulted in excellent disease control, acceptable toxicity, and very low rates of vaginal/pelvic failure. The 5-year RFS and OS were $85 \%$ and $93 \%$, respectively. The 2-year vaginal RFS was $98 \%$. These results do not appear inferior to those of standard-dose brachytherapy (18-21 Gy in 3 fractions prescribed at $0.5 \mathrm{~cm}$, surface EQD2 54 Gy) [9-12,15,17], and appear comparable to that achieved with other low-dose schema (24 Gy in 6 fractions, prescribed to vaginal surface, surface EQD2 28 Gy) [13].

For endometrial cancer patients with endometrioid histology, there is evidence and growing interest in the concept that a lower vaginal surface dose may be as effective as the higher "classic" brachytherapy prescription dose. For example, one randomized trial from Sweden of 290 low-risk endometrial patients showed that excellent
Table 1. Patient and tumor characteristics $(n=56)$

\begin{tabular}{|c|c|c|}
\hline Characteristic & Level & $\%(n)$ \\
\hline Age & Median (range) & $66(47-81)$ \\
\hline Age $\geq 70$ & Yes & $32(18)$ \\
\hline Race & $\begin{array}{c}\text { Caucasian } \\
\text { African American } \\
\text { Unknown/Other }\end{array}$ & $\begin{array}{l}70(39) \\
20(11) \\
10(6)\end{array}$ \\
\hline Obese $(\mathrm{BMI} \geq 30)$ & Yes & $53(29)$ \\
\hline Smoking history & Yes & $22(12)$ \\
\hline Oral contraceptive history & Yes & $36(19)$ \\
\hline $\begin{array}{l}\text { History of hormone replace- } \\
\text { ment therapy }\end{array}$ & Yes & $6(3)$ \\
\hline Family history of cancer & Yes & $40(22)$ \\
\hline Histology & $\begin{array}{l}\text { Mixed USC/Other } \\
\text { Pure uterine } \\
\text { serous carcinoma }\end{array}$ & $\begin{array}{l}40(22) \\
60(34)\end{array}$ \\
\hline $\begin{array}{l}\text { Endocervical glandular } \\
\text { involvement }\end{array}$ & Yes & $16(9)$ \\
\hline $\begin{array}{l}\text { Endocervical stromal } \\
\text { involvement }\end{array}$ & Yes & $9(5)$ \\
\hline Myometrial invasion $\geq 50 \%$ & Yes & $21(12)$ \\
\hline Stage - FIGO 2009 & $\begin{array}{l}\text { IA } \\
\text { IB } \\
\text { II }\end{array}$ & $\begin{array}{c}73(41) \\
18(10) \\
9(5) \\
\end{array}$ \\
\hline Stage - FIGO 1988 & $\begin{array}{l}\mathrm{A} \\
\mathrm{IB} \\
\mathrm{IC} \\
\mathrm{I} A \\
\| \mathrm{B} \\
\mathrm{II} \mathrm{A}\end{array}$ & $\begin{array}{c}25(13) \\
41(22) \\
18(10) \\
7(4) \\
9(5) \\
4(2) \\
\end{array}$ \\
\hline $\begin{array}{l}\text { Lymph-vascular space } \\
\text { invasion }\end{array}$ & Yes & $29(16)$ \\
\hline $\begin{array}{l}\text { Lower uterine segment } \\
\text { involvement }\end{array}$ & Yes & $38(20)$ \\
\hline Cytology positive & Yes & $4(2)$ \\
\hline
\end{tabular}

rates of vaginal control were achievable with six fractions of $2.5 \mathrm{~Gy}$ prescribed to $5 \mathrm{~mm}$ from the applicator surface (surface EQD2 30 Gy) [18]. A randomized study sponsored by the PORTEC group (PORTEC-4) is investigating whether three fractions of 5 Gy prescribed at $5 \mathrm{~mm}$ (surface EQD2 $\sim 33 \mathrm{~Gy}$ ) is non-inferior to three fractions of 7 Gy (surface EQD2 54 Gy) [19]. However, patients with USC are specifically excluded from this protocol. We believe that further study of alternate dose schema in USC patients receiving chemotherapy is worthwhile. The incidence of grade 1-2 vaginal toxicity, including atrophy, shortening, and stenosis is approximately 35\% when prescribing standard dose brachytherapy [19]. Concomitant chemotherapy may increase the incidence of vaginal dryness and discomfort in patients undergoing IVB [20], and furthermore, sexual dysfunction can be related to changes in body image associated with chemotherapy-induced menopause and hair loss. Therefore, lowering the dose to the vaginal mucosa in patients re- 


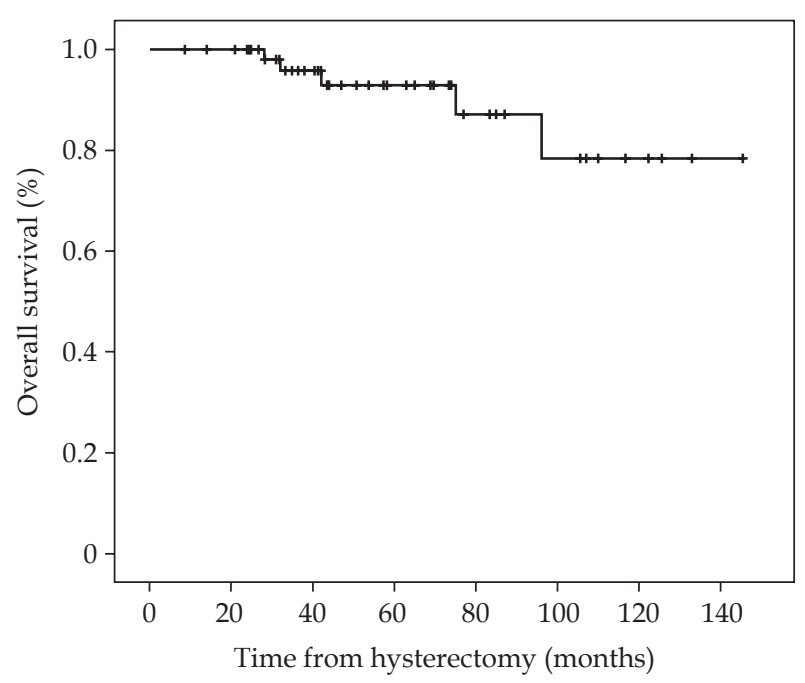

Fig. 1. Overall survival - stage I-II uterine serous carcinoma $(n=56)$

ceiving chemotherapy may improve toxicity, however, to our knowledge, this concept has not yet been studied.

In the present series, there were no grade 3-4 vaginal toxicities, and grade 2 vaginal toxicities among the 56 patients were recorded in only two patients $(3.6 \%)$, which is remarkably low, and compares favorably to the literature. However, it must be noted that due to the retrospective nature of this report, toxicity recording was not standardized, and thus grade 1-2 toxicities were likely under-reported. To truly assess a benefit of this lower dose regimen compared to standard-dose brachytherapy, particularly concerning vaginal toxicities, prospective, standardized recording of toxicities is required. This is a topic being investigated prospectively at our institution [21].

Table 2. Patient/tumor characteristics and univariate log-rank results for overall (OS) and relapse-free survival (RFS)

\begin{tabular}{lcc} 
Characteristic & $\begin{array}{c}\text { OS, } \\
p \text {-value }\end{array}$ & $\begin{array}{c}\text { RFS, } \\
\text {-value }\end{array}$ \\
\hline Age $(\geq 70$ vs. $<70)$ & 0.062 & 0.132 \\
\hline Race (Caucasian vs. other) & 0.716 & 0.388 \\
\hline Obese $(\mathrm{BMI} \geq 30$ vs. $<30)$ & 0.249 & 0.281 \\
\hline Smoking history & 0.008 & 0.076 \\
\hline Oral contraceptive history & 0.634 & 0.182 \\
\hline History of hormone replacement therapy & 0.575 & 0.226 \\
\hline Family history of cancer & 0.502 & 0.579 \\
\hline Histology (pure USC vs. mixed) & 0.168 & 0.551 \\
\hline Endocervical glandular involvement & 0.530 & 0.278 \\
\hline Endocervical stromal involvement & 0.450 & 0.768 \\
\hline Myometrial invasion $(\geq 50 \%$ vs. $<50 \%)$ & 0.001 & $<0.0001$ \\
\hline Lymph-vascular space invasion & 0.971 & 0.003 \\
\hline Lower uterine segment involvement & 0.388 & 0.551 \\
\hline Cytology positive & 0.716 & 0.587
\end{tabular}

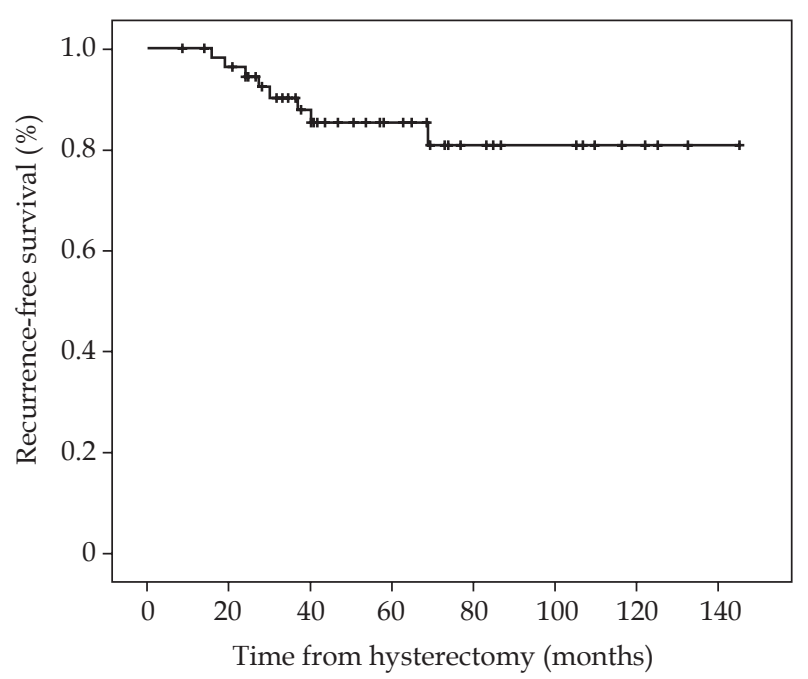

Fig. 2. Recurrence-free survival - stage I-II uterine serous carcinoma $(n=56)$

The pattern of recurrence of USC differs from that of its endometrioid counterpart, and abdominal/peritoneal recurrence is described in even clinical stage I disease [1-2]. Support for platinum/taxane chemotherapy regimens with or without RT is mounting [5-7]. Critics of the use of RT in early stage USC might wonder, if two IVB fractions are as good as three, why not abandon RT completely. However, eliminating RT in early stage USC appears to result in very high rates of vaginal recurrence $[5,22,23]$. In one study of 74 stage I USC patients who were surgically staged, none of 43 patients who received radiation to the vaginal cuff recurred locally, however, six of the $31(19 \%)$ patients who were not treated with vaginal radiation recurred at the vaginal cuff [5]. In another report of 60 USC (and other unfavorable histology) patients who had adjuvant radiation therapy, one had a recurrence at the vaginal apex, whereas of the 65 patients who did not have adjuvant radiation, 8 had a local-regional recurrence, which included 3 with a vaginal only recurrence, 1 vaginal, and pelvic recurrence and 4 had pelvic recurrence [22]. Further, in an investigation of the pattern of failure among 23 early stage USC patients, of those treated without $\mathrm{RT}$, five of nine failures were within the pelvis, of which 4 relapsed in the vagina; however, there were no pelvic failures in women treated with adjuvant RT [23]. Thus, although the optimal IVB dose fractionation scheme for treatment of patients with early stage USC is not known, the current available data do support the importance of multi-modality therapy including comprehensive surgery [24], chemotherapy [6,7], and RT [5,22,23].

There is a lack of clear evidence concerning the preferred radiotherapy approach for USC; thus adjuvant radiotherapy practices for early stage USC continue to vary by country and by institution. Some believe that IVB alone may be inadequate due to risk of non-vaginal pelvic recurrences, and therefore tumor-directed pelvic RT in combination with chemotherapy is practiced by many. For example, at MD Andersen Cancer Center, pelvic radiotherapy in combination with paclitaxel resulted 
Table 3. Stage I-II uterine serous carcinoma pattern of recurrence

\begin{tabular}{cccccccc}
$\begin{array}{c}\text { Patient } \\
\text { number }\end{array}$ & $\begin{array}{c}\text { Age at } \\
\text { diagnosis }\end{array}$ & $\begin{array}{c}\text { Stage } \\
- \text { FIGO } \\
2009\end{array}$ & $\begin{array}{c}\text { Stage } \\
- \text { FIGO } \\
1988\end{array}$ & $\begin{array}{c}\text { Myometrial } \\
\text { invasion } \\
(\geq 50 \%)\end{array}$ & LVI & $\begin{array}{c}\text { Site of first recurrence } \\
\text { surgery to } \\
\text { recurrence } \\
\text { (months) }\end{array}$ \\
\hline 1 & 72 & IA & IIA & - & + & Pericardium, cervical and supraclavicular nodes & 27 \\
\hline 2 & 74 & IA & IA & - & N/A & Lung, colon, and abdominal wall \\
\hline 3 & 63 & IB & IC & + & + & Lung \\
\hline 4 & 81 & IB & IC & + & + & Lung and vagina & 16 \\
\hline 5 & 80 & IB & IC & + & - & Liver and lung & 24 \\
\hline 7 & 67 & IB & IC & + & + & Peri-aortic nodes & 30 \\
\hline 8 & 51 & IB & IC & + & + & Peri-aortic nodes and lung & 69 \\
\hline 64 & II & IIB & + & - & Lung, liver, carcinomatosis
\end{tabular}

in 5-year PFS of $83 \%$ among stage I-IIIA USC patients [25]. Yet other institutions have supported IVB in favor of pelvic RT due to its favorable side effect profile and the predilection of USC for distant relapse. For example, at Memorial Sloan-Kettering Cancer Center, a retrospective analysis of 77 early stage USC patients treated with IVB without pelvic RT (majority also receiving chemotherapy) found 5-year pelvic recurrence of $6 \%$. These authors concluded that this rate was not high enough to justify routine use of pelvic RT in this population [26]. More insight into optimal radiotherapy and chemotherapy combinations for this rare disease, both with respect to disease control as well as toxicity, will likely emerge once data mature from the recently accrued GOG 249 (pelvic RT versus IVB + chemo) and ongoing PORTEC-3 (pelvic RT versus pelvic RT + chemo) trials, both of which include USC patients within the study populations.

While the results of our early stage USC cohort were excellent overall, the majority of patients $(73 \%)$ had stage IA disease. On closer analysis, stage IB patients appear to fare less well, with a 5 -year RFS of $47 \%$ for those with deep myometrial invasion. The association of stage IB with relatively high risk of extra-pelvic failures is in agreement with multiple other studies $[8,24,26,27]$. A recent population-based study from British Columbia, which studied pelvic RT in combination with 3 cycles of $\mathrm{C} / \mathrm{T}$ chemotherapy [27] found a 5-year disease-free survival of $48.5 \%$ for patients with stage IB disease, and a majority of the recurrences were extra-pelvic. The application of more targeted therapies to treat USC, even in uterine-confined disease with deep myometrial invasion, may be needed.

In our series, there was a single patient who had experienced a vaginal failure. This patient had numerous risk factors for recurrence including $>50 \%$ depth myometrial invasion and lymph-vascular space involvement, as well as simultaneous lung metastases, as shown in Table 3. Whether the vaginal relapse may have been prevented had a higher dose of brachytherapy and/or pelvic radiotherapy been utilized is not known. Overall, the 2-year vaginal failure rate of $2 \%$ in this series appears comparable to that of similar cohorts of early stage USC and/or high-risk endometrial patients, which have used a median dose of 21-Gy in 3 fractions, and reported 2-5 year vag- inal failure rates of $2-4 \%$ [10-12,15,26]. Direct comparison, however, is not possible given the heterogeneity of stages (percentage stage IA versus IB) and histologies (pure USC versus mixed)

Our study has several important limitations. It is not randomized, and there is no comparison group. In addition, the cohort of patients studied is small, although comparable to other publications reporting on this rare disease. It is important to note that all patients in this series were treated with comprehensive aggressive staging by experienced gynecologic oncologists, and therefore this regimen may not be satisfactory for women who have had less aggressive surgery. Furthermore, the population described had all received $\mathrm{C} / \mathrm{T}$ chemotherapy, and hence the use of 2 fractions of $7 \mathrm{~Gy}$ as described in this report may not be appropriate for women with other chemotherapy regimens, fewer than 6 cycles of chemotherapy, or non-serous histology. Moreover, given that, a majority of patients in this series $(79 \%)$ had $<50 \%$ myometrial invasion, our findings may not be generalizable to patients with stage IB or higher disease. Also, caution should be used in applying this regimen when using cylinder sizes smaller than $2.6 \mathrm{~cm}$, due to the relatively high fractional surface dose to the mucosa with this regimen. Lastly, given the retrospective nature of the study, information on vaginal toxicity was not recorded consistently. An ongoing prospective investigation at our institution examines whether this reduced-dose IVB regimen is associated with improved toxicity compared to more standard IVB schema.

\section{Conclusions}

Debate continues concerning the preferred radiotherapy approach for early stage USC. In our series, when combined with contemporary chemotherapy, adjuvant HDR IVB delivered as 2 fractions of 7 Gy prescribed at a depth of $0.5 \mathrm{~cm}$ resulted in excellent vaginal/pelvic control rates for localized USC. Further study of brachytherapy technique in USC investigating the optimal dose, number of fractions, time interval between fractions, and when to schedule with chemotherapy is warranted. 


\section{Disclosure}

Authors report no conflict of interest.

\section{References}

1. Hendrickson M, Ross J, Eifel P et al. Uterine papillary serous carcinoma: a highly malignant form of endometrial adenocarcinoma. The Am J Surg Pathol 1982; 6: 93-108.

2. Boruta DM, 2nd, Gehrig PA, Fader AN et al. Management of women with uterine papillary serous cancer: a Society of Gynecologic Oncology (SGO) review. Gynecol Oncol 2009; 115: 142-153.

3. Sutton G, Axelrod JH, Bundy BN et al. Adjuvant whole abdominal irradiation in clinical stages I and II papillary serous or clear cell carcinoma of the endometrium: a phase II study of the Gynecologic Oncology Group. Gynecol Oncol 2006; 100 : 349-354.

4. Martinez AA, Weiner S, Podratz K et al. Improved outcome at 10 years for serous-papillary/clear cell or high-risk endometrial cancer patients treated by adjuvant high-dose whole abdomino-pelvic irradiation. Gynecol Oncol 2003; 90: 537-546.

5. Kelly MG, O'Malley DM, Hui P et al. Improved survival in surgical stage I patients with uterine papillary serous carcinoma (UPSC) treated with adjuvant platinum-based chemotherapy. Gynecol Oncol 2005; 98: 353-359.

6. Goldberg H, Miller RC, Abdah-Bortnyak R et al. Outcome after combined modality treatment for uterine papillary serous carcinoma: a study by the Rare Cancer Network (RCN). Gynecol Oncol 2008; 108: 298-305.

7. Fader AN, Drake RD, O'Malley DM et al. Platinum/taxanebased chemotherapy with or without radiation therapy favorably impacts survival outcomes in stage I uterine papillary serous carcinoma. Cancer 2009; 115: 2119-2127.

8. Turner BC, Knisely JP, Kacinski BM et al. Effective treatment of stage I uterine papillary serous carcinoma with high doserate vaginal apex radiation (192Ir) and chemotherapy. Int J Rad Oncol Biol Phys 1998; 40: 77-84.

9. Alektiar KM, Makker V, Abu-Rustum NR et al. Concurrent carboplatin/paclitaxel and intravaginal radiation in surgical stage I-II serous endometrial cancer. Gynecol Oncol 2009; 112: 142-145.

10. Kiess AP, Damast S, Makker V et al. Five-year outcomes of adjuvant carboplatin/paclitaxel chemotherapy and intravaginal radiation for stage I-II papillary serous endometrial cancer. Gynecol Oncol 2012; 127: 321-325

11. Barney BM, Petersen IA, Mariani A et al. The role of vaginal brachytherapy in the treatment of surgical stage I papillary serous or clear cell endometrial cancer. Int J Rad Oncol Biol Phys 2013; 85: 109-115.

12. Eldredge-Hindy HB, Eastwick G, Anne PR et al. Adjuvant vaginal cuff brachytherapy for high-risk, early stage endometrial cancer. J Contemp Brachytherapy 2014; 6: 262-270.

13. Townamchai K, Berkowitz R, Bhagwat M et al. Vaginal brachytherapy for early stage uterine papillary serous and clear cell endometrial cancer. Gynecol Oncol 2013; 129: 18-21.

14. Small W, Jr., Beriwal S, Demanes DJ et al. American Brachytherapy Society consensus guidelines for adjuvant vaginal cuff brachytherapy after hysterectomy. Brachytherapy 2012; 11: 58-67.

15. Landrum LM, Nugent EK, Zuna RE et al. Phase II trial of vaginal cuff brachytherapy followed by chemotherapy in early stage endometrial cancer patients with high-intermediate risk factors. Gynecol Oncol 2014; 132: 50-54.

16. Nag S, Gupta N. A simple method of obtaining equivalent doses for use in HDR brachytherapy. Int J Rad Oncol Biol Phys 2000; 46: 507-513.
17. DuBeshter B, Estler K, Altobelli K et al. High-dose rate brachytherapy for Stage I/II papillary serous or clear cell endometrial cancer. Gynecol Oncol 2004; 94: 383-386.

18. Sorbe B, Straumits A, Karlsson L. Intravaginal high-dose-rate brachytherapy for stage I endometrial cancer: a randomized study of two dose-per-fraction levels. Int J Rad Oncol Biol Phys 2005; 62: 1385-1389.

19. https://www.msbi.nl/promise/Projects/PORTEC4.aspx, last accessed 03/02/2014

20. Vakilian S, Al-Suhaibani, A, Bahoric B. What causes vaginal stenosis in patients undergoing adjuvant intracavitary brachytherapy for endometrial cancer? Proceedings of the $53^{\text {rd }}$ Annual ASTRO Meeting. Abstract 2553. Int J Rad Oncol Biol Phys 2011; 81: S475.

21. Park HS, Ratner E, Lucarelli L et al. Predictors of vaginal stenosis following intra-vaginal high-dose-rate brachytherapy for endometrial carcinoma. Proceedings of the $55^{\text {th }}$ Annual ASTRO Meeting. Abstract 240. Int J Rad Oncol Biol Phys 2014; 90: S111.

22. Ly D, Rowley BD, Dodson MK et al. Adjuvant radiation in early stage, unfavorable histology endometrial carcinoma is associated with improved local control and survival. Gynecol Oncol 2014; 133: 250-255.

23. Mehta N, Yamada SD, Rotmensch J et al. Outcome and pattern of failure in pathologic stage I-II papillary serous carcinoma of the endometrium: implications for adjuvant radiation therapy. Int J Rad Oncol Biol Phys 2003; 57: 1004-1009.

24. Slomovitz BM, Burke TW, Eifel PJ et al. Uterine papillary serous carcinoma (UPSC): a single institution review of 129 cases. Gynecol Oncol 2003; 91: 463-469.

25. Jhingran A, Ramondetta LM, Bodurka DC et al. A prospective phase II study of chemoradiation followed by adjuvant chemotherapy for FIGO stage I-IIIA (1988) uterine papillary serous carcinoma of the endometrium. Gynecol Oncol 2013; 129: 304-309.

26. Desai NB, Kiess AP, Kollmeier MA et al. Patterns of relapse in stage I-II uterine papillary serous carcinoma treated with adjuvant intravaginal radiation (IVRT) with or without chemotherapy. Gynecol Oncol 2013; 131: 604-608.

27. van der Putten LJ, Hoskins P, Tinker A et al. Populationbased treatment and outcomes of Stage I uterine serous carcinoma. Gynecol Oncol 2014; 132: 61-64. 